\title{
SPECTROSCOPIC STUDIES OF EMISSION LINE GALAXIES
}

\author{
M. S. CHUN, E. C. SUNG and H. K. MOON \\ Department of Astronomy and Atmospheric Science, \\ Yonsei University, Seoul, Korea \\ and \\ Y. I. BYUN \\ Institute for Astronomy, University of Hawaii, \\ Honolulu, Hawaii, U.S.A.
}

\begin{abstract}
.
Spectroscopic Observations were made to study 42 emission line objects. The analysis of these long slit spectra shows that 15 out of 42 galaxies are blue compact galaxies (BCGs). 21 of them are starforming or HII galaxies and 3 were found to be normal galaxies.
\end{abstract}

\section{Observation}

We observed emission line objects during July 8-14, 1991 and April 3-8, 1992. The Mount Stromlo Observatory(MSO) 74-inch telescope equipped with the spectrograph and Photon Counting Array(PCA) was used during these observational runs. The spectral range covered $3,500-5,500 \AA$ and $4,800-7,000 \AA$.

\section{Data Reduction and Results}

We used NOAO. IRAF 2.0 package for data reduction in the standard way. We listed identifications of these objects in TABLE 1 . Among the 42 objects, 9 galaxies show the spectra of typical starburst galaxy. The radial velocity difference between ESO 513-IG11 and ESO $513-$ G10 is found to be $250 \mathrm{~km} / \mathrm{sec}$, and it is conceivable that they consist of an interacting system as a faint patch between the two galaxies can be seen on ESO red plate. ESO 105-IG11 is thought to be an iE type blue compact galaxy which has two highly excited HII regions in the nucleus and faint outer envelope of an elliptical shape.

T. J.-L. Courvoisier and A. Blecha: Multi-Wavelength Continuum Emission of AGN, 442-443.

(C) 1994 IAU. Printed in the Netherlands. 
TABLE I

Identifications of the Galaxies

\begin{tabular}{|c|c|c|c|}
\hline object & identifications & object & identifications \\
\hline ESO 122-IG02 & starburst galaxy & ESO 270-IG22 & starburst galaxy(?) \\
\hline 124-I12 & starburst galaxy & CTS 1033 & BCG \\
\hline 060-IG03 & starburst galaxy & 1034 & HII galaxy \\
\hline 036-IG03 & no emission lines & ESO 386-G09 & Seyfert galaxy(?) \\
\hline 566-IG08 & HII galaxy & 513-IG11 a & BCG \\
\hline 435-G20 & BCG & 513-IG11 b & BCG \\
\hline CTS 1010 & HII galaxy & 513-IG11 c & spiral galaxy \\
\hline 1011 & BCG & 042-IG04 & BCG or HII galaxy \\
\hline 1012 & BCG & CTS 1037 & BCG \\
\hline ESO 436-IG42 & starburst or HII galaxy & ESO 102-G14 & BCG, high excitation \\
\hline 264-IG13 & interacting galaxy & 140-G09 a & BCG or HII galaxy \\
\hline CTS 1020 & BCG & $140-G 09 \mathrm{~b}$ & BCG or HII galaxy \\
\hline ESO $376-I G 17$ & starburst galaxy & 281-G07 & HII region \\
\hline 502-IG11 a & BCG & 338-IG04 & BCG, interacting \\
\hline 502-IG11 b & BCG & 338-IG08 & starburst galaxy \\
\hline UM 448 & BCG or HII galaxy & 105-IG11 & BCG, high excitation \\
\hline 462 & BCG & 342-IG13 & not BCG, weak [OII] \\
\hline ESO 505-G12 & BCG or HII galaxy & $530-\mathrm{G} 42$ & no emission lines(?) \\
\hline CTS 1027 & BCG & 289-IG08 & starburst galaxy \\
\hline ESO 322-IG32 & starburst galaxy & 290-G01 & starburst galaxy \\
\hline 383-G20 & not $\mathrm{BCG}$, strong[NI] & 293-G04 & irregular galaxy \\
\hline
\end{tabular}

